\title{
Extreme and critical transition events in the memristor based Liénard system
}

\author{
S. Leo Kingston ${ }^{1,2, a}$, K. Suresh ${ }^{3}$, K. Thamilmaran ${ }^{1}$, and T. Kapitaniak ${ }^{2}$ \\ ${ }^{1}$ Department of Nonlinear Dynamics, School of Physics, Bharathidasan University, \\ Tiruchirappalli 620024, Tamil Nadu, India \\ 2 Division of Dynamics, Lodz University of Technology, Stefanowskiego 1/15, \\ 90-924 Lodz, Poland \\ ${ }^{3}$ Department of Physics and Astrophysics, University of Delhi, Delhi 110007, India
}

Received 9 August 2019 / Received in final form 20 September 2019 Published online 26 March 2020

\begin{abstract}
We study extreme and critical events in the forced Liénard systems with charge control memristor. It has been found that the system exhibits hidden attractors either in the absence or presence of an external sinusoidal force. We give evidence that these attractors play a crucial role in the appearance of critical events. We attempt to explain the mechanism leading to the emergence of catastrophic transitions. Finally, we present that the observed critical transitions are typical for memristor based models and understanding of them gives some insight on how to avoid these types of devastating events at the time of the device fabrication process.
\end{abstract}

\section{Introduction}

Studies on extreme events (EEs) received notable attention in the last few decays owing to their catastrophic impact on nature and society [1]. These EEs are occurring in an unexpected way, which causes disastrous consequences. Understanding and predicting EEs would have considerable value for societies such as predicting events like droughts, flooding, seasonal changes, climate forecasting for agriculture, and financial crashes. However, we do not know all the relevant mechanisms and generic character of EEs and many researchers are focusing on various reliable techniques [2] to understand EEs. Understanding the origin of EEs would help us to predict and anticipate them. In recent years emergence of extreme events has been reported in different disciplines [1-7]. In two coupled and inhomogeneous units of FitzHugh Nagumo model, interior crisis plays a crucial role in the generation of extreme events [4]. The appearance of extreme events via three different routes namely period doubling, quasiperiodic and intermittency has been studied in the forced Liénard system and Hindmarsh Rose neuron model [5,6]. Similarly, a new class of extreme events has been identified in dissipative dynamical systems with discontinuous boundaries, where extreme events arise due to stick-slip dynamics [7]. For more understanding of extreme events and to explore its different mechanism, in this study we focus on extreme and critical transition events in the memristor based driven Liénard system.

a e-mail: kingston.cnld@gmail.com 
For that, we have taken charge control memristor using the smooth cubic nonlinear function.

The concept of memristor coined by the circuit theorist Leon O Chua in 1971, explains the relation between charge $(q)$ and flux $(\phi)$ of the missing fourth fundamental circuit elements [8]. The remarkable property of the memristor is both resistive and memory-like nature. Besides the resistance of the memristor depends on the past history of input voltage or current, it can function as synapses of the neuron. The most important applications of this element are nonvolatile memory and neuromorphic computing. In very recent years, neuromorphic computing devices received significant attention, since it has an appealing structure for artificial synapses [9]. Memristor device is not commercially available till date. To address this lacuna, for the better understanding of its own properties and their interaction with the other circuit elements, memristor emulator circuits were proposed in the literature $[10,11]$. Presently, these memristor emulators are used for different artificial synapses such as metaplasticity [12], nociceptor [13], and resistive switching behavior [14]. Furthermore, there are several researchers in material science laboratories endeavor to realize this device using $\mathrm{CuO}[15], \mathrm{Au} / \mathrm{TiO}_{2} / \mathrm{Au}$ [16], Tantalum oxide [17] and Silver chalcogenide [18] based materials. The memristor emulator circuits exhibit the pinched hysteresis loop in the voltage versus the current plane. There are different nonlinearities used to describe the charge and flux relationships of the memristor emulator circuits, such as nonsmooth piecewise linearity, smooth cubic nonlinearity, and smooth piecewise quadratic nonlinearity [19].

The memristor based Liénard system exhibits multiple periodic or chaotic attractors for the fixed system parameters with the different choice of initial conditions. Nevertheless, these attractors are not connected with any fixed points of the system and located away from the fixed point, these attractors known as hidden attractors. The well familiar nonlinear models such as van der Pol, Rössler, Chua's circuit, Belousov-Zhabotinsky, and many other systems exhibit periodic or chaotic attractors for the typical choice of system parameters. These attractors located as the neighborhood of its unstable fixed point are denoted as self excited attractors. If the basin attractions of an attractor do not associate with its neighborhood of any fixed point they are denoted as hidden attractors [20]. Recently, this interesting topic of research is relighted by Leonov and his research group in different nonlinear systems $[20,21]$. The rare chaotic flow with hidden attractors is reported in [22], it explains significant three possibilities of the emergence of hidden attractors in complex systems such as, without fixed points, with the line of fixed point and from the stable equilibrium point. Presently, researchers are in the different field of science interested in revealing these hidden attractors in memristor based nonlinear system for possible applications [23-26].

Memristor based hyperchaotic system manifests coexisting of infinite numbers of hidden attractors and transient hyperchaos [24]. The autonomous memristor based Duffing oscillator shows line invariance of infinite numbers of hidden attractors [23]. When introducing the memristor in standard nonlinear models such as Lorenz and Rössler oscillators, the fixed point of the system disappeared, which leads to the emergence of hidden attractors. This is an important observation since the above mentioned systems widely used for several engineering applications [27]. The intuition of the appearance of hidden attractors and their different properties helps us to understand some undesired behaviors. Moreover, this is an essential study for analyzing sudden switching to unexpected attractors. Hence these sudden shifts lead to the diversity of catastrophic events such as financial crisis, disasters in commercial devices, and abrupt climate changing processes [20,28].

In the present study, we explain the emergence of EEs in the memristor based forced Liénard system for the suitable choice of system parameters. Nevertheless, the 
system exhibits critical transition events which show non-Gaussian probability distribution function it represents that atypical dynamical transition. The structure of this paper presented as follows, Section 2 explains the model system and its detailed stability analysis. The emergence of extreme events in the memristor based Liénard system is illustrated in Section 3. Section 4 deals with the existence of critical transition events and hidden attractors in the considered model. The final conclusion explored in Section 5.

\section{Model system}

The passive two-terminal memristor devices have a functional relationship between charge $(q)$ and flux $(\phi)$. Its terminal voltage $(v)$ and current $(i)$ described by the relation $v=M(q) i$ and $i=W(\phi) v$, where $v=\frac{d \phi}{d t}$ and $i=\frac{d q}{d t}$. Here $M(q)$ and $W(\phi)$ are the different nonlinear function denoted as memristance and memductance, respectively, which are defined as $M(q)=\frac{d \phi(q)}{d q}$ and $W(\phi)=\frac{d q(\phi)}{\phi}$. For this study, we have taken the charge control memristor $M(q)$, using the smooth cubic nonlinearity which is defined as $\phi(q)=\omega_{0}^{2} q+\beta q^{3}$. Further, we have used the same model for realizing the smooth cubic nonlinearity as reported in [19]. To study the influence of charge control memristor in a nonlinear system and explore its different complex dynamics, we have taken the following form of Liénard system $[5,29]$

$$
\ddot{x}+\alpha x \dot{x}+\omega_{0}^{2} x+\beta x^{3}=0 .
$$

Equation (1) satisfied the general form of Liénard system as $\ddot{x}+f(x) \dot{x}+g(x)=0$, here $f(x)=\alpha x$ and $g(x)=\omega_{0}^{2} x+\beta x^{3}$. This Liénard system is paradigmatic model for different disciplines such as physics, engineering, chemistry and biological system which shows diverse of rich dynamics $[5,29,30]$. There are distinct advantages that have been explained in the chemical and biochemical kinetic equation when it model as Liénard-type chemical oscillator [31]. The resonant tunneling diode based optoelectronic oscillator is represented as Liénard system using external perturbation and time delay feedback [32]. In this study to analyze the memristor effect in equation (1), we consider $g(x)$ as memristor function $M(q) x$ and include external periodic force in the system. Hence the memristor based forced Liénard system is represented by the following equation

$$
\ddot{x}+\alpha x \dot{x}+M(q) x=f \sin (\omega t)
$$

where $\alpha x, M(q) x$, and $f \sin (\omega t)$ are the position depended damping coefficient, memristor function, and external forcing respectively. When we include the memristor function in equation (2), an additional dimension increased in the system. The memristor function expressed as $M(q)=\frac{d \phi(q)}{d q}=\omega_{0}^{2}+3 \beta q^{2}$. For numerical study, we used the variables $q=x_{1}, x=x_{2}$ and $\dot{x}=x_{3}$ and equation (2) can be written as the following form of first order equations,

$$
\begin{aligned}
& \dot{x_{1}}=x_{2} \\
& \dot{x_{2}}=x_{3} \\
& \dot{x_{3}}=-\alpha x_{2} x_{3}-\left(\omega_{0}^{2}+3 \beta x_{1}^{2}\right) x_{2}+f \sin (\omega t) .
\end{aligned}
$$

Equation (3) are solved numerically using the fourth order Runge-Kutta algorithm with the fixed step size $h=2 \pi / 1024 \omega$. First we analyze the dynamics of unforced system $(f=0.0)$. For that the system parameters are fixed as $\alpha=0.45, \beta=0.17$ and $\omega_{0}^{2}=-0.45$, which exhibits periodic attractor. Throughout this paper for studying 


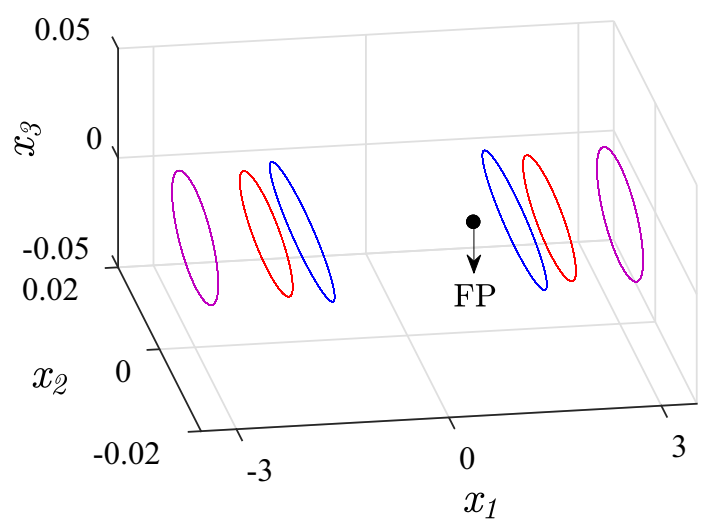

Fig. 1. Coexistence of multiple hidden attractors for the system parameter values as $\alpha=$ $0.45, \beta=0.17$ and $\omega_{0}^{2}=-0.45$ and FP refers the fixed point of the system.

different complex dynamics, we have taken $\omega_{0}^{2}<0$, since this parameter plays virtual role for the emergence of different dynamics in the system. A detailed stability analysis for equation (3) is given in the appendix.

Based on the stability analysis, the equilibrium point is in the saddle state for the parameters value of $\alpha=0.45, \beta=0.17$ and $\omega_{0}^{2}=-0.45$. However, the system exhibits multiple periodic attractors for the different initial conditions. These attractors are so-called hidden attractors (HA), since these attractors do not approach its fixed point and are located away from the equilibrium point. The coexistence of multiple periodic hidden attractors for the unforced system is shown in Figure 1. It can be noticed from the figure that attractors are not approaching the fixed point of the system and are situated away from the equilibrium point. In the following section, we will discuss how these HA are behaving when we include external force in the system.

\section{Extreme events}

In this section, we explain the emergence of extreme events in the memristor based Liénard system with external forcing. The system exhibits extreme events for the parameters $\alpha=0.3, \beta=1.0, \omega_{0}^{2}=-1.68, f=0.4$ and $\omega=0.349$. To show the appearance of extreme events, we have plotted the peaks of the time series $x_{3}$ in Figure 2a. The time series of Figure 2a denoted as extreme events because it signifies the large amplitude oscillations from its bounded motion which are rare but recurrent events. Note that most of the time system oscillate the maximum amplitude of $\approx 3.5$. In Figure 2a, the horizontal red line represents the significant height $\left(H_{S}\right)$ threshold. In general, the occurrence of extreme events in different models is confirmed by the statistical measurement of significant height $\left(H_{S}\right)$ threshold criteria [33,34]. Here we consider $H_{S}$ value as mean $\left(\left\langle P_{n}\right\rangle\right)$ plus six times of standard deviation $(\sigma)\left(H_{S}=\right.$ $\left.\left\langle P_{n}\right\rangle+6 \sigma\right)$ for peaks of the time the series $\left(P_{n}\right)$.

We determine the probability distribution function (PDF) for peaks $\left(P_{n}\right)$ of one of the system state variables $x_{3}$ is shown in Figure 2b. The PDF portrays long-tail distribution, which confirms the occurrence of extreme events [29], it also crosses the significant height $\left(H_{S}\right)$ (marked as the vertical dashed line in Fig. 2b). Remarkably, the appearance of these extreme events is highly sensitive depending on its initial conditions. To confirm this, we have plotted the phase diagram with respect to its two different initial conditions as shown in Figure 3. The existence of extreme events (red 

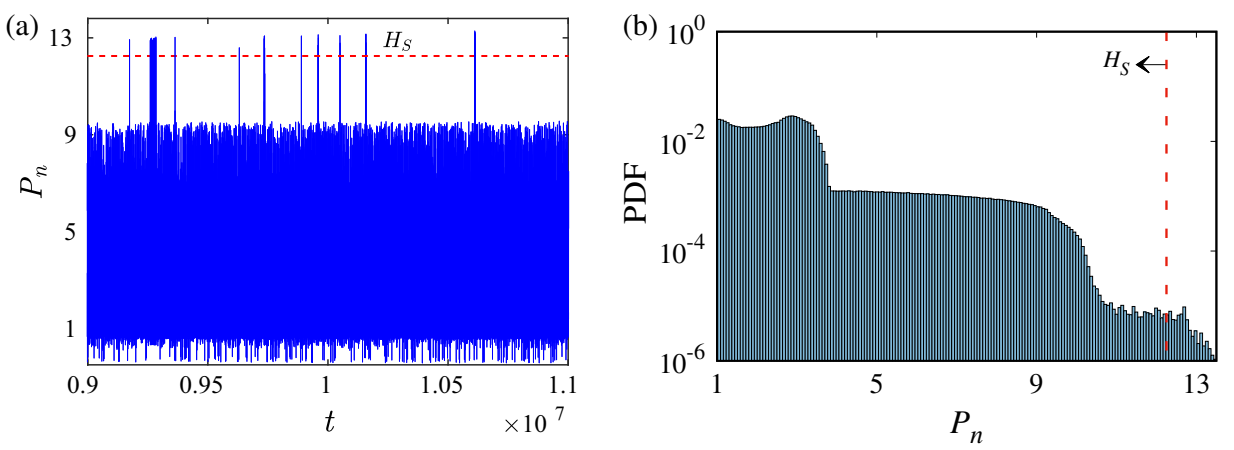

Fig. 2. (a) Time series of extreme events and the horizontal red line signifies the extreme event qualifier threshold $\left(H_{S}\right)$. (b) Probability distribution function represents the fat tail distribution which is also exceed the significant height $\left(H_{S}\right)$.

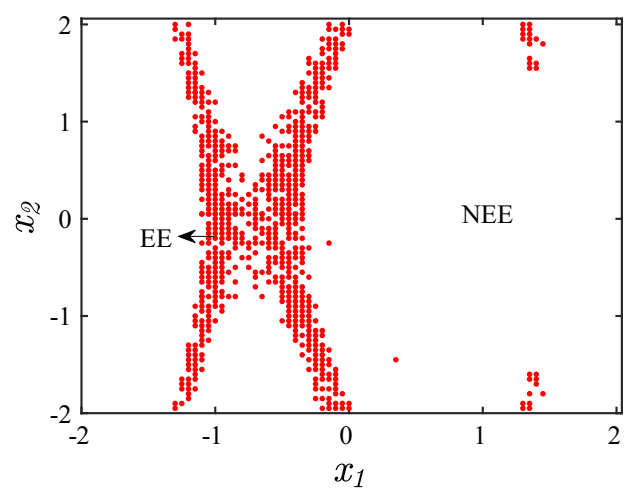

Fig. 3. Phase diagram to distinguish the existence of extreme events (EE - red points) and nonextreme events (NEE - white regions) for fixed system parameters as $\alpha=0.3$, $\beta=1.0, \omega_{0}^{2}=-1.68, f=0.4$ and $\omega=0.349$ by varying the initial conditions in the typical parameters phase.

points) and nonextreme event (white regions) is discriminated based on the extreme event qualifier threshold criteria. We noticed from the phase diagram, the existence of extreme events in this system is rare since it arises only in the small region as compared with the large area of nonextreme event regions.

\section{Critical transition event}

In addition to the extreme events, the memristor based forced Liénard system exhibits critical transition events for the another choice of system parameters values as $\alpha=$ $0.45, \beta=0.17, \omega_{0}^{2}=-0.45, f=0.4$ and $\omega=0.185$. To portray the critical transition event, we have plotted only the peaks of the time series of $x_{3}$, which is represented in Figure 4a. Interestingly it shows an astonishing transition in the asymptotic state of the system. The aperiodic oscillations are switched to quasiperiodic motion through the unusual large amplitude oscillation. Finally the quasiperiodic motion is settled in to regular periodic state (for clear visualization we shown the stable periodic attractor in the insert of Figure 4a). The emergence of this phenomenon and the appearance of the time interval of aperiodic oscillations in the system are sensitive depending on initial conditions, which we will explain later in the next subsection. Further, we 

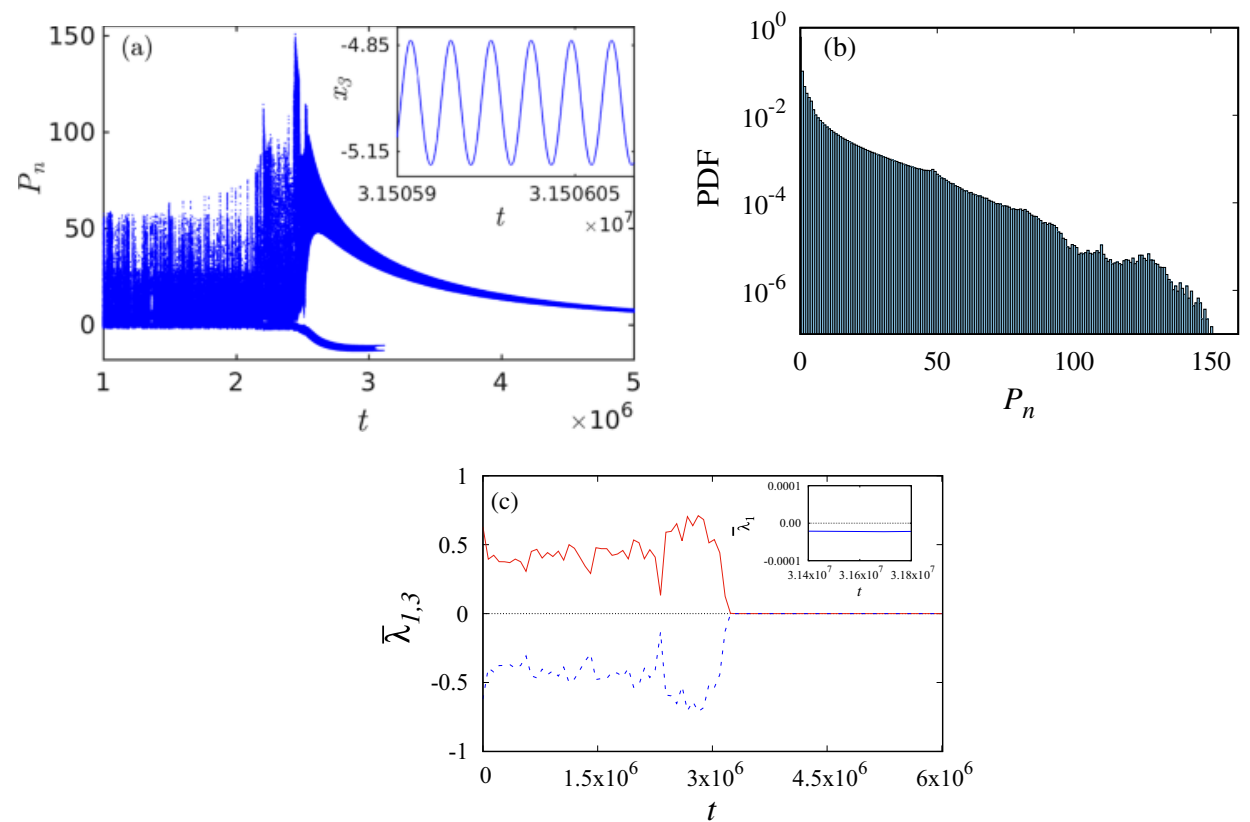

Fig. 4. (a) Peaks of time series $\left(P_{n}\right)$ represent the critical transition events and the insert shows periodic oscillations. (b) Probability distribution function for long run of peaks of the time series signifies non-Gaussian distribution. (c) Finite time Lyapunov exponent represents the transition of system from aperiodic state to quasiperiodic motion.

plotted the PDF for this critical transition event as shown in Figure 4b. For plotting the PDF, we have taken the extremely long run of peaks of the time series, which shows the non-Gaussian PDF. This non-Gaussian probability distribution function is one of the significant properties to confirm the existence of several catastrophic transitions as reported in $[35,36]$. We understand from Figure $4 \mathrm{~b}$, due to the unusual large amplitude events the system shows non-Gaussian PDF.

The statistical analysis of finite time Lyapunov exponent (FTLE) is used to examine the amount of stretching or folding of the system state variables, over the finite time segments along a given trajectory $[37,38]$. We have used equation (3) to compute the FTLE. The Lyapunov exponent is calculated

$$
\Lambda^{m}=\lim _{N \rightarrow \infty} \frac{1}{N} \sum_{i=1}^{N} \log \left\|\mathbf{e}_{i}^{m}\right\|, \quad m=1,2, \ldots k .
$$

Here the $\mathbf{e}_{i}^{m}$ is the reorthonormalized vectors and are given

$$
\mathbf{e}_{i}^{m}=\mathbf{J F}\left(x_{1_{i}}, x_{2_{i}}, x_{3_{i}}\right) \cdot \hat{\mathbf{e}}_{i-1}^{m}
$$

where the $\mathbf{J F}$ represent the Jacobian matrix and the $i$ refer time step. The finite time Lyapunov exponent is defined as

$$
\bar{\lambda}_{j}^{m}=\frac{1}{M} \sum_{j=1}^{M} \lambda_{j}^{m}, \quad m=1,2, \ldots k .
$$

In the equation (6) $M$ denotes a finite time interval and $\lambda_{j}^{m}$ is the instantaneous Lyapunov exponent $\left(\lambda_{j}^{m}=\log \left\|\mathbf{e}_{i}^{m}\right\|\right)$. For the present study we have used the finite 

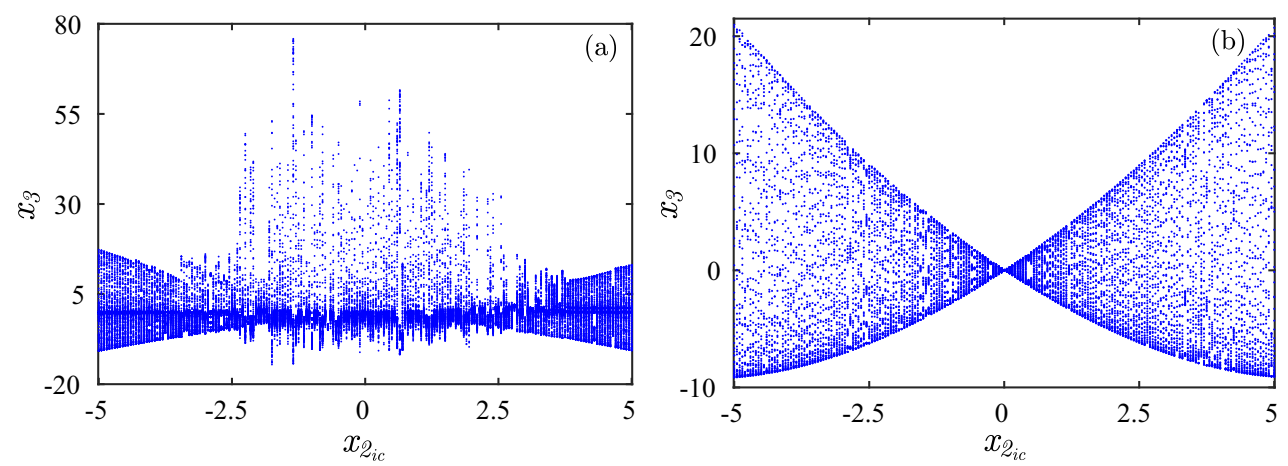

Fig. 5. Bifurcation diagram to represent the coexistence of different attractors for fixed system parameters and varying initial conditions. (a) Existence aperiodic and quasiperiodic attractors and (b) only quasiperiodic motion for the typical range of initial conditions.

time $M=500$. In order to identify different transitions in the time series, we have plotted the FTLE distribution as shown in Figure 4c. The maximum Lyapunov spectra for the finite time interval denoted as the red-solid line in Figure 4c, whereas the blue-dashed line represents the third Lyapunov spectra of the system. The maximum Lyapunov spectra are positive at the beginning, it signifies that the system is in the chaotic state. After a very long excursion, the system transits to quasiperiodic motion (cf. Fig. 4a) represented by zero Lyapunov spectra in Figure 4c. Finally, the trajectory settle into the periodic state shows negative maximum Lyapunov exponent in the insert of Figure 4c. Hence, the FTLE spectra confirm the existence of different dynamics in the time series.

\subsection{Multistability}

Moreover, for the same parameters values of critical transition event, with different initial conditions, the system exhibits complete quasiperiodic motion. It reveals that the coexistence of different attractors of the system such as critical transition events and quasiperiodic motion, these coexistence of multiple attractors are in a system, denoted as multistability [39]. In order to confirm the existence of multistability nature in memristor based Liénard system, one parameter bifurcation diagram was plotted by fixing the parameters value as $\alpha=0.45, \beta=0.17, \omega_{0}^{2}=-0.45, f=0.4$ and $\omega=0.185$ and varying the initial conditions for the typical range as shown in Figure 5, where $x_{2_{i c}}$ represents the initial conditions for the system state variable $x_{2}$. Figure 5a shows the coexistence of critical transition events and quasiperiodic attractors for the fixed initial conditions $x_{1}=-0.1, x_{3}=0.01$ and varying $x_{2_{i c}} \in$ $(-5,5)$. However, for another set of initial conditions as $x_{1}=-5.0, x_{3}=0.01$ and varying $x_{2}$ in the range $-5.0 \leq x_{2_{i c}} \leq 5.0$, the system shows only quasiperiodic motion which is represented in Figure $5 \mathrm{~b}$.

Furthermore, we are trying to locate the regions of critical transition events and quasiperiodic attractors with respect to its initial conditions. For that, the basin of attractions is plotted using the fixed system parameters and varying its two different initial conditions as shown in Figure 6, with two different projections. In the basin of attraction plots of Figure 6, the gray and blue regions signify quasiperiodic and chaotic states, respectively. Interestingly, these quasiperiodic motions are hidden attractors, because its basin does not approach the fixed points of the system, which we will explain elaborately in the next subsection. 

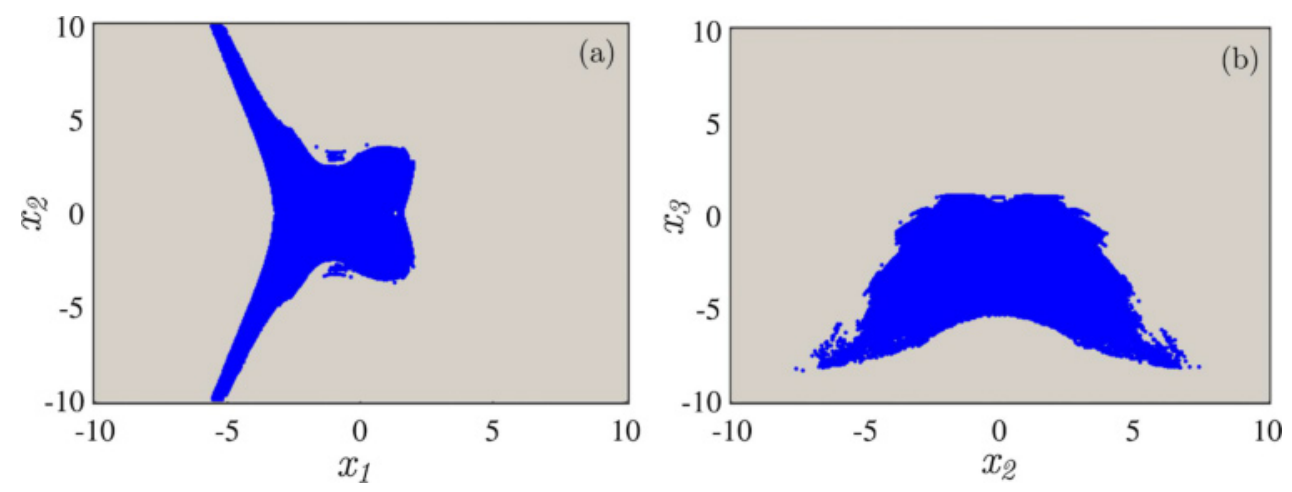

Fig. 6. Basin of attraction (a) in the $\left(x_{1}-x_{2}\right)$ and (b) $\left(x_{2}-x_{3}\right)$ planes, for the fixed system parameters as $\alpha=0.45, \beta=0.17, \omega_{0}^{2}=-0.45, f=0.4$ and $\omega=0.185$. The two different regions such as quasiperiodic (gray) and chaotic (blue) states are discriminated based on the Lyapunov stability.

(a)

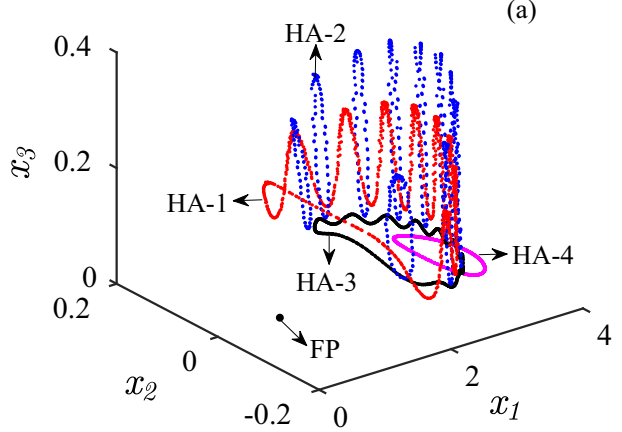

(b)

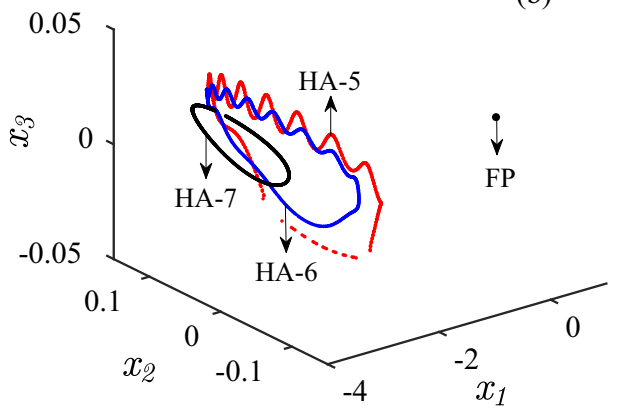

Fig. 7. Coexistence of hidden attractors: Poincaré surface of section for quasiperiodic motion, where initial are conditions chosen in the (a) positive $x_{1}$ axis and (b) negative $x_{1}$ axis. FP denotes the fixed point of the system, and all the attractors located away from the fixed point of the system.

\subsection{Hidden attractors in driven Liénard system}

As we mentioned earlier, the memristor based Liénard system also exhibits hidden attractors with the presence of external forcing. For that the parameter values of the equation (3) fixed as $\alpha=0.45, \beta=0.17, \omega_{0}^{2}=-0.45, f=0.4$ and $\omega=0.185$, the system portrays infinite number of hidden attractors with respect to different initial conditions. To prove the coexistence of several hidden attractors of quasiperiodic motion with different initial conditions, we display its Poincaré surface of section as shown in Figures 7a and 7b. It's clear that all the hidden attractors are placed away from its fixed points. The advent of this hidden attractor plays an important role in different dynamical evaluation processes in critical transition events.

It is worthwhile to investigate the relationship between critical transition events and hidden attractors in the memristor based Liénard system. To perform this, we plotted the peaks of two different time series of hidden attractors $\left(H_{1}-\right.$ green and $H_{2}$ - blue) using the specific interval of time as shown in Figure 8a. In Figure 8a, the time series critical transition event ( $\mathrm{CTE}$ - red) taken as the particular regions (that is only the small part of its quasiperiodic motion) after the very long transient. Time series of hidden attractors superimposed with the same time interval of critical 

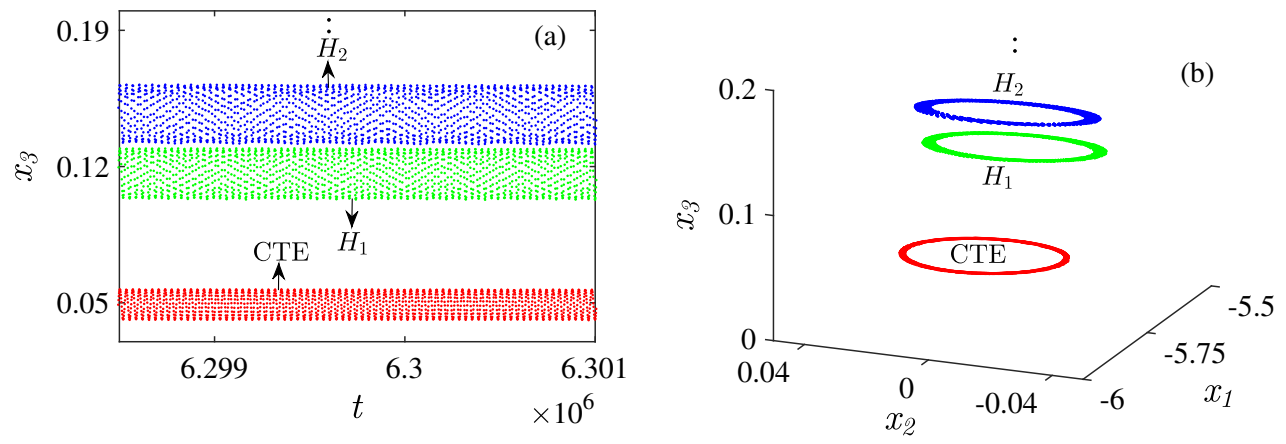

Fig. 8. (a) Time series of critical transition events (CTE - red) which is superimposed with two different hidden attractors $\left(H_{1}-\right.$ green and $H_{2}-$ blue $)$ denoted that the quasiperiodic motion of critical transition events settled in to the hidden attractors and (b) its corresponding phase portraits in the three dimensional plane.

transition events. This result denotes that after the large amplitude excursion the quasiperiodic motions of critical transition event merges with hidden attractors. It is clearly shown in the time series plots of state variable $x_{3}(t)$ (Fig. 8a), and its corresponding three dimensional phase portraits shown in Figure 8b. However, the detailed emerging mechanism of critical transition events is left for future work. The above investigation emphasizes that understanding of hidden attractors will help to explore several catastrophic transitions in the diversity of models which used in different disciplines. Notably, the deep knowledge of this catastrophic transition in engineering models will help to given precaution, before the occurrence of damage in the commercial devices.

\section{Conclusion}

We studied the extreme and critical transition events in the memristor based driven Liénard system. The chosen model exhibits hidden attractors in the presence as well as the absence of external forcing. The obtained extreme events were confirmed by significant height threshold criteria, and also it shows long-tail probability distribution function. The critical transient events show non-Gaussian probability distribution function due to the large amplitude excursions and finite time Lyapunov exponent confirms the different dynamical transitions in the time series. The advent of critical transition events and hidden attractors is a sensitive dependence on its initial conditions, which verified by using one parameter bifurcation diagram, and basin of attractions. Notably, the obtained hidden attractors in the forced Liénard system play an essential role in the existence of different critical transition dynamics in the system. This observation would give some intuition to understand various catastrophic transitions in different materials and natural events.

S.L.K. and T.K. have been supported by the Polish National Science Centre, MAESTRO Programme-Project No. 2013/08/A/ST8/00780. K.S. is supported by the UGC, Government of India through Dr D.S. Kothari Postdoctoral Fellowship. K.T. acknowledges DSTPURSE, Govt. of India for financial support. 

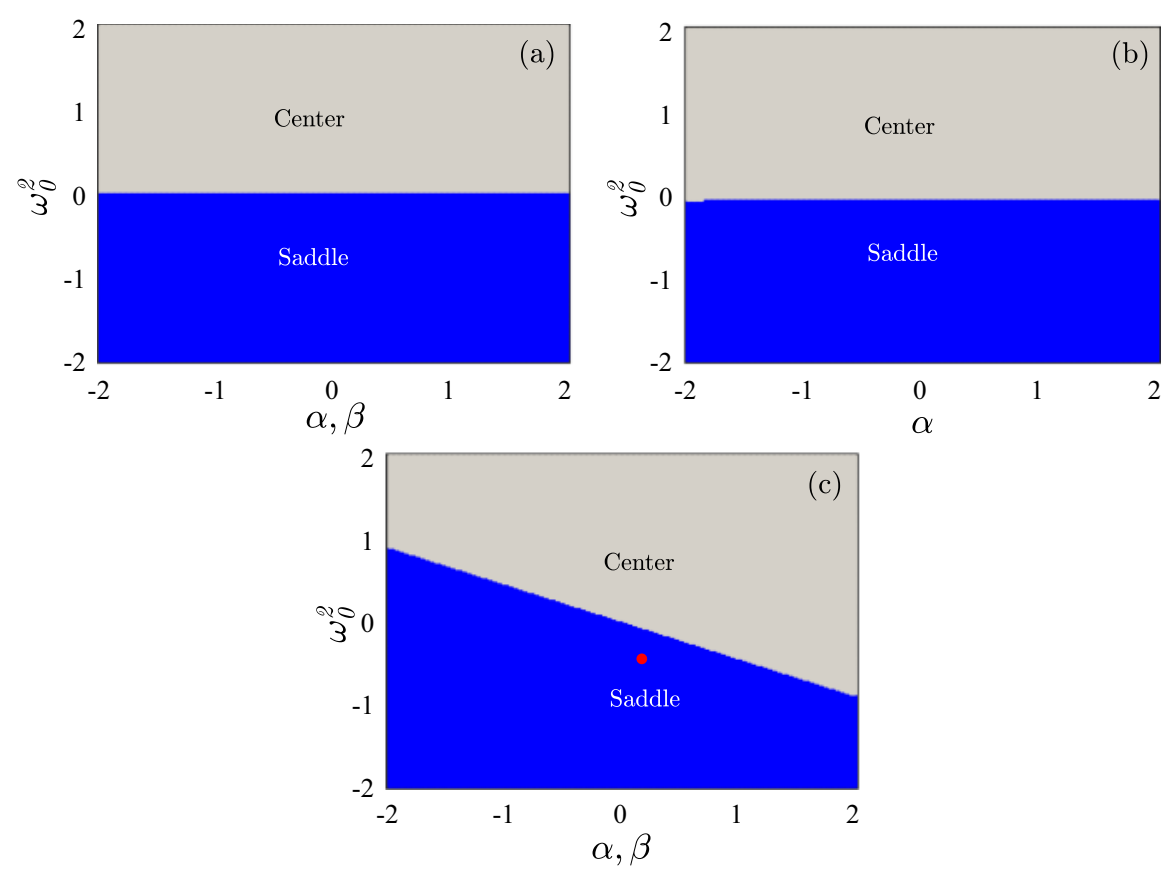

Fig. 9. Stability phase diagrams of unforced Liénard system equation (3) for different parameter region, (a) dissimilar stability regions for zero $x^{*}$ values, where as (b) and (c) represents the stability regions for nonzero $x^{*}$ values.

\section{Author contribution statement}

S.L.K. performed the numerical simulations. S.L.K. and K.S. wrote the first draft of the manuscript. All the authors equally contributed in manuscript preparation and revisions.

Open Access This is an open access article distributed under the terms of the Creative Commons Attribution License (https://creativecommons.org/licenses/by/4.0), which permits unrestricted use, distribution, and reproduction in any medium, provided the original work is properly cited.

\section{Appendix A: Stability analysis}

We analyzed the stability of the system equation (3), in the absence of external forcing. The equilibrium points of the system is calculated by using $\dot{x_{1}}=\dot{x_{2}}=\dot{x_{3}}=0$, and the obtained equilibrium points are $\left(x_{1}^{*}, 0,0\right)$. In that, the fixed point value of $x_{1}^{*}$ can be either zero or nonzero. For calculating eigenvalues, the Jacobian matrix for the system equation (3) in the absence of external forcing term written as

$$
\operatorname{det}(M-\lambda I)=\left(\begin{array}{ccc}
0-\lambda & 1 & 0 \\
0 & 0-\lambda & 1 \\
0 & -\alpha x_{3}-\omega_{0}^{2}-3 \beta x_{1}^{* 2}-\alpha x_{2}-\lambda
\end{array}\right)=0
$$

The characteristic equation for the above matrix is

$$
\left(\alpha x_{3}\right) \lambda^{3}+\left(\alpha x_{2}\right) \lambda^{2}+\left(\alpha x_{3}+\omega_{0}^{2}+3 \beta x_{1}^{2}\right) \lambda=0
$$


and the eigenvalues for the equation (A.2) is

$$
\begin{aligned}
\lambda_{1} & =0 \\
\lambda_{2,3} & =\frac{1}{2}\left[-\alpha x_{2} \pm \sqrt{\alpha^{2} x_{1}^{2}-4 \alpha x_{3}-4 \omega_{0}^{2}-12 \beta x_{1}^{* 2}}\right] .
\end{aligned}
$$

The stability of the system at $\left(\dot{x_{1}}, \dot{x_{2}}, \dot{x_{3}}\right)=(0,0,0)$ is depends on the value of $\omega_{0}^{2}$, (i.e.) the system is in the Center type for $\omega_{0}^{2}>0$, whereas for $\omega_{0}^{2}<0$ the stability of system is in Saddle state which shown in Figure 9a with different parameter regions. Besides for nonzero $x_{1}^{*}$ value, the system exhibits the same stability like zero $x_{1}^{*}$ value case, such as Center and Saddle respectively. However, its existing stability regions differ with respect to the different parameter regions. The stability phase diagram for nonzero $x_{1}^{*}$ values is shown in Figures $9 \mathrm{~b}$ and $9 \mathrm{c}$. When including the external sinusoidal forcing into the system, we obtained the same eigenvalues as given in equation (A.3). For the parameter values of $\alpha=0.45, \beta=0.17$ and $\omega_{0}^{2}=-0.45$, the system is in the saddle state which represented as red point in Figure 9c.

\section{References}

1. S. Albeverio, V. Jentsch, H. Kantz, Extreme events in nature and society (Springer, Heidelberg, 2005)

2. N.M. Alvarez, S. Borkar, C. Masoller, Eur. Phys. J. Special Topics 226, 1971 (2017)

3. A.N. Pisarchik, R. Jaimes-Retegui, R. Sevilla-Escoboza, G. Huerta-Cuellar, M. Taki, Phys. Rev. Lett. 107, 274101 (2011)

4. R. Karnatak, G. Ansmann, U. Feudel, K. Lehnertz, Phys. Rev. E 90, 022917 (2014)

5. S.L. Kingston, K. Thamilmaran, P. Pal, U. Feudel, S.K. Dana, Phys. Rev. E 96, 052204 (2017)

6. A. Mishra, S. Saha, M. Vigneshwaran, P. Pal, T. Kapitaniak, S.K. Dana, Phys. Rev. E 97, 062311 (2018)

7. K. Suresh, A.N. Pisarchik, Phys. Rev. E 98, 032203 (2018)

8. L. Chua, IEEE Trans. Circuit Theory 18, 507 (1971)

9. Y. Park, J.S. Lee, ACS Nano 11, 8962 (2017)

10. H. Kim, M.P. Sah, C. Yang, S. Cho, L.O. Chua, IEEE Trans. Circuits Syst. I 59, 2422 (2012)

11. A. Adamatzky, L.O. Chua, Memristor networks (Springer, London, 2013)

12. Q. Wu, H. Wang, Q. Luo, W. Banerjee, J. Cao, X. Zhang, F. Wu, Q. Liu, L. Li, M. Liu, Nanoscale 10, 5875 (2018)

13. J.H. Yoon, Z. Wang, K.M. Kim, H. Wu, V. Ravichandran, Q. Xia, C.S. Hwang, J.J. Yang, Nat. Commun. 9, 417 (2018)

14. S. Kim, H. Kim, S. Hwang, M.H. Kim, Y.F. Chang, B.G. Park, ACS Appl. Mater. Interfaces 9, $40420(2017)$

15. T.D. Dongale, P.S. Pawar, R.S. Tikke, N.B. Mullani, V.B. Patil, A.M. Teli, K.V. Khot, S.V. Mohite, A.A. Bagade, V.S. Kumbhar, K.Y. Rajpure, P.N. Bhosale, R.K. Kamat, P.S. Patil, J. Nanosci. Nanotechnol. 18, 984 (2018)

16. H.A. Bafrani, M. Ebrahimi, S.B. Shouraki, A.Z. Moshfegh, Nanotechnology 29, 015205 (2018)

17. A.C. Torrezan, J.P. Strachan, G. Medeiros-Ribeiro, R.S. Williams, Nanotechnology 22, $485203(2011)$

18. A.S. Oblea, A. Timilsina, D. Moore, K.A. Campbell, Proc. Int. Jt. Conf. Neural Netw. 3, $4(2010)$

19. S. Sabarathinam, C.K. Volos, K. Thamilmaran, Nonlinear Dyn. 87, 37 (2017)

20. D. Dudkowski, S. Jafari, T. Kapitaniak, N. Kuznetsov, G. Leonov, A. Prasad, Phys. Rep. 637, 1 (2016)

21. G. Leonov, N. Kuznetsov, V. Vagaitsev, Physica D 241, 1482 (2012) 
22. S. Jafari, J. Sprott, F. Nazarimehr, Eur. Phys. J. Special Topics 224, 1469 (2015)

23. V. Varshney, S. Sabarathinam, A. Prasad, K. Thamilmaran, Int. J. Bifurc. Chaos 28, $1850013(2018)$

24. B. Bao, H. Bao, N. Wang, M. Chen, Q. Xu, Chaos Solitons Fractals 94, 102 (2017)

25. Q. Xu, Y. Lin, B. Bao, M. Chen, Chaos Solitons Fractals 83, 186 (2016)

26. M. Chen, M. Li, Q. Yu, B. Bao, Q. Xu, J. Wang, Nonlinear Dyn. 81, 215 (2015)

27. P. Saha, D. Saha, A. Ray, A. Chowdhury, Eur. Phys. J. Special Topics 224, 1563 (2015)

28. M. Scheffer, J. Bascompte, W. Brock, V. Brovkin, S. Carpenter, V. Dakos, H. Held, E.H van Nes, M. Rietkerk, G. Sugihara, Nature 461, 53 (2009)

29. S.L. Kingston, K. Thamilmaran, Int. J. Bifurc. Chaos 27, 1730025 (2017)

30. S.L. Kingston, K. Suresh, K. Thamilmaran, AIP Conf. Proc. 1942, 060008 (2018)

31. S. Ghosh, D.S. Ray, Eur. Phys. J. B 87, 65 (2014)

32. B. Romeira, J. Figueiredo, C.N. Ironside, J. Javaloyes, in Dynamics of Liénard optoelectronic oscillators, selected topics in nonlinear dynamics and theoretical electrical engineering (Springer-Verlag, Berlin, 2013), pp. 117-136

33. E. Mercier, A. Even, E. Mirisola, D. Wolfersberger, M. Sciamanna, Phys. Rev. E 91, $042914(2015)$

34. J. Reinoso, J. Zamora-Munt, C. Masoller, Phys. Rev. E 87, 062913 (2013)

35. A. Montina, U. Bortolozzo, S. Residori, F.T. Arecchi, Phys. Rev. Lett. 103, 173901 (2009)

36. P. Walczak, S. Randoux, P. Suret, Phys. Rev. Lett. 114, 143903 (2015)

37. K. Suresh, A. Prasad, K. Thamilmaran, Phys. Lett. A 377, 612 (2013)

38. A. Prasad, R. Ramaswamy, Phys. Rev. E 60, 2761 (1999)

39. U. Feudel, Int. J. Bifurc. Chaos 18, 1607 (2008) 